\title{
Prognostic value of neutrophil-to-lymphocyte ratio and location in patients with EGFR mutant metastatic non-small cell lung cancer treated with TKIs
}

\section{Xueqi Xie}

Shandong First Medical University

\section{Xiaolin Li}

Shandong Cancer Hospital and Institute

\section{Wenjie Tang}

University of Jinnan Shandong Academy of Medical Sciences

Jinlong Chen

Shandong Cancer Hsptal and Institute

Minghuan Li

Shandong Cancer Hospital and Institute

Peng Xie ( $\nabla$ xiepengro@126.com )

Shandong Tumor Hospital and Institute https://orcid.org/0000-0001-8647-2882

Jinming Yu

Shandong Cancer Hospital and Institute

Research article

Keywords: Epidermal growth factor, NSCLC, TKI, Primary location, NLR, PFS

Posted Date: November 3rd, 2020

DOI: https://doi.org/10.21203/rs.3.rs-29351/v3

License: (9) (i) This work is licensed under a Creative Commons Attribution 4.0 International License. Read Full License 


\section{Abstract}

Background: Targeted therapy with the epidermal growth factor receptor (EGFR) tyrosine kinase inhibitors (TKIs) has improved the field of metastatic non-small cell lung cancer treatment. Higher neutrophil-tolymphocyte ratio (NLR) and lower relative lymphocyte counts as inflammatory indicators and associated with worse overall survival and progression free survival (PFS) in several tumor types. Few studies focused on these inflammation markers in context of TKIs eras.

Methods: Patients with advanced EGFR mutation NSCLC treated with TKIs were included. Pre-treatment NLR means neutrophil to lymphocyte ratio measured in peripheral blood within one week before treating with TKIs. The baseline clinical characteristics of each group were compared by chi-square and $t$ tests. Cox regression analyses were used to evaluate prognostic value of peripheral blood parameters on progression free survival (PFS). All prognostic factors were explored with multivariable regression.

Results: We retrospectively analyzed 221 patients with metastatic NSCLC harboring exon 19 deletion, 21 L858R or rare mutation and receiving TKIs. Finally, a total of 190 patients were analyzed. The optimal cutoff values for pretreatment absolute lymphocyte count (Lym), lymphocyte percentage (Lym\%), absolute neutrophil count (Neu), the percentage of neutrophil granulocytes (Neu\%) and NLR were $1.625 \mathrm{~B}$, $18.8 \%, 3.675 a, 51.8 \%$ and 4.965 , respectively. Patients with high neutrophil percent (13.0 months vs 18.8 months, $\mathrm{P}=0.003)$, absolute neutrophil counts (12.0 months vs 14.5 months, $\mathrm{P}=0.014)$ and NLR (7.0 months vs 15.2 months, $P<0.001$, one-year PFS Rate, 55.3\%, respectively) had worse PFS. In contrast, patients with high absolute lymphocyte counts (13.0 months vs 16.5 months, $\mathrm{P}=0.012$ ) and lymphocyte percent ( 8.8 months vs 15.3 months, $P<0.001$ ) had a better PFS. Besides, tumor location was also an important factor for prognosis ( 11.6 months vs 14.3 months, $\mathrm{P}=0.003$ ). On multivariate analysis, NLR and primary tumor location were both identified as independent and significantly risk indicators for worse PFS.

Conclusion: NLR and primary location are both independent prognostic factors for PFS in patients with metastatic EGFR mutated lung tumor. Whether or not NLR and primary location could be useful markers in efficacy prediction of TKIs in advanced NSCLC calls for further investigation.

\section{Background}

Lung cancer continues to be leading causes of cancer-related deaths in the world, especially patients with metastatic stage [1]. Non-small cell lung cancer (NSCLC) as the most common type of lung carcinoma, accounts for approximately $85 \%-90 \%$ of all lung cancer cases. Lung neoplasm can also be subgrouped into central and peripheral types according to primary tumor location [2]. Pulmonary adenocarcinoma pathological subtypes have replaced squamous cell carcinoma in recent years, often located at peripheral, as one of the most common cell type of lung cancer. In recent years, a number of previous 
studies have shown that primary location is an important factor to guide treatment schedules and predict clinical prognosis in lung tumors[3-6].

The advances in epidermal growth factor receptor tyrosine kinase inhibitors EGFR-TKIs of non-small cell lung cancer (NSCLC) have led to a new area of target therapy, particular in patients with NSCLC who have EGFR mutation. Until recent, targeted therapy remains the first-line treatment for the majority of patients with targetable oncogenic driver alterations. In recent years, gefitinib, erlotinib, afatinib, and osimertinib have showed better clinical outcomes and responses rates compared with chemotherapy using cytotoxic drugs.

Host immunity may affect prognosis in patients with various cancers. Evidence have demonstrated that immune system plays an important role in promoting antitumor defense. Tumor-associated inflammation and tumor microenvironment play a critical role in cancer development, progression and metastasis[7-9]. The neutrophil-to-lymphocyte ratio (NLR) is one of the most widely used inflammation biomarkers in solid cancer that can be easily calculated from routine blood examination results. Inflammation not only can contribute to development of various cancers, but also recognized as a hallmark of cancer[10]. For example, several peripheral blood parameters-including markers of systemic inflammation such as baseline the neutrophil-to-lymphocyte ratio (NLR), the lymphocyte-to-monocyte ratio (LMR) and the absolute lymphocyte count have been associated with survival in patients with metastatic lung neoplasm treated with the ICls[11, 12].

Neutrophil counts, lymphocyte counts and NLR as the significant roles in the inflammatory response also demonstrates its value in various of solid tumors. As people costs much in treatment, a cheaper, readily and more effective potential prognostic markers need to be assisted the prognosis and patient risk stratification of the lung cancer. The previous studies showed that the NLR can be considered as a predictor to evaluate the prognosis, which can be used in the EGFR-TKIs and ICls [13-15]. Few studies find the association between NLR and OS. There is a lack of understanding the relationship between peripheral blood counts and PFS, particularly, in patients treated with TKIs.

The aim of our study was to find the prognostic value of pretreatment complete blood count (CBC) parameters in NSCLC patients treated with EGFR-TKIs in advanced NSCLC as from first line to third line. Additionally, we needed to find various determined factors to predict clinical outcomes. In general, the main objective was to explore more effective, useful and noninvasive predictors to assess the probability of patients receiving benefit from TKls treatment.

\section{Methods}

\subsection{Patients and clinical characteristics}

This retrospective study was approved by review board of Shandong cancer hospital and institute. We respectively analyzed patients with metastatic or recurrent postoperative NSCLC. All patients needed to meet the following standard criteria: 1) were of 18 years or older, 2) pathologically proven 
adenocarcinoma with a positive EGFR mutation test before any treatment, including chemotherapy, surgery, radiotherapy and targeted therapy, 3) complete medical record/CT scans of the chest and/or PET scans/bronchoscopy, 4) treatment with TKI drugs as the first line, second line or third line, 5) complete peripheral blood test results within 1 week before receiving EGFR-TKI treatment, including neutrophil and lymphocyte counts, 6) TKI drugs included Gefitinib, Erlotinib and Loctinib. Thus, patients who meet all the above-mentioned criteria were included from the electronic record system.

The clinical stage was determined by the 7th edition of the AJCC/UICC staging system. Pre-treatment NLR means that the nearest peripheral absolute neutrophils and absolute lymphocytes ratio before starting of TKIs within 1 week. PFS was defined as from the time treated with the EGFR-TKIs to the terminology or pathology evidence of progression or recurrence. The OS was measured from the day of proving NSCLC to the date of death from any courses.

\subsection{EGFR Mutation Test}

A total of 190 specimens before EGFR-TKIs therapies, were obtained via tissue biopsy, including bronchoscopy, CT-guided biopsy, or surgical procedures from primary or metastatic sites. If we cannot obtain the tissues, we would take from peripheral blood and metastatic body fluids. The majority of samples use the peptide nucleic acid (PNA)-mediated PCR clamping method. Few samples were sequenced with targeted next generation sequencing (NGS) of 18 lung cancer gene panel.

\subsection{Tumor location evaluation}

According to patients' CT imaging, bronchoscopy, or both methods, we identified into central and peripheral lung cancer. As there is no standard definition to classify peripheral and central lung tumor. We defined central tumors as occurring from segmental or proximal bronchi. As for peripheral type, we considered that tumors in subsegmental or other distal bronchi and bronchioli according to previous studies. ${ }^{12}$ All images were anonymized and blindly evaluated by one radiologist and one oncologist. For discordant cases, we would assign the third oncologist.

\section{Statistical analysis}

Pre-treatment blood data were obtained from electronic medical records. Student's t-test was used to evaluate the difference in absolute lymphocyte counts, relative lymphocyte counts and relative neutrophil level in two different groups. We use Man-Whitney t-test for the ordinal data and Pearson's $\chi 2$ or Fisher's exact test to compare clinical characteristics at baseline. EGFR mutation and primary location (central vs. peripheral) were analyzed using chi-square tests. Receiver operating characteristic (ROC) cure analysis was used to determine the optimal value of absolute lymphocyte count, percentage of the lymphocyte count, the absolute neutrophil count, the percentage of neutrophil counts and the Neutrophil-toLymphocyte ratio in terms of their association with PFS. Patients and clinical characteristic were summarized with descriptive statistics. We also use the Kaplan-Meier method and log-rank for univariate survival analysis. A Cox regression was used for multivariate analysis of the different clinical 
characteristics on PFS and OS, which was done in Statistical analyses were performed by SPSS 19.0. $\mathrm{P}<0.05$ was recognized statistically significant.

\section{Results}

\subsection{Patients and tumor characteristics}

From January 2014 to November 2018, a total of consecutive 221 patients were treated with TKIs. 31 patients were not meet the eligible standards (29 patients missed complete peripheral blood tests, 1 patient's image data was hard to define primary tumor location and 1 patient missed image data before TKIs). Baseline characteristics of the patients are summarized in table 1 . The majority of patients were female ( $\mathrm{N}=122)$, median age was 58.0 (range: 30 - 87 years), and 148 patients had never smoking habits. 172 patients underwent PCR test and 18 underwent NGS test. We also found the most oncogenic alterations were EGFR L858R mutant $(\mathrm{N}=110)$. Additionally, eighty-three patients had bone metastasis (43.7\%), and 26 (13.7\%) liver metastasis and 59 (31.1\%) had brain metastasis. Besides, many patients received chemotherapy as the first-line therapy $(\mathrm{N}=90)$. 27 patients undergo surgery before target therapies. No correlations were found between primary location and EGFR mutation status. (Chi-Square Tests $=0.76, \mathrm{P}=0.963) .142$ patients are peripheral-type adenocarcinoma. The number of stage IV patients were 164. The patients in postoperative recurrence were 26.

\subsection{Optimal cutoff value for complete blood count}

Median PFS was 12.51 months. When we use PFS as an end point for blood routine. According to ROC analysis, we obtained the following optimal cutoff values: 1.625 for pre-treatment Lym, $18.8 \%$ for pretreatment Lym\%, 3.675 for pre-treatment Neu, $51.8 \%$ for pre-treatment Neu\% and 4.965 for pre-treatment NLR for PFS (Table 3). Among the many peripheral blood routine indicators, pre-treatment Lym has the largest AUC, which is $63.2 \%$. However, the pre-treatment Neu\% has the lowest AUC, which is $49.9 \%$, indicating its low predictive value.

\subsection{Association between pre-treatment complete blood count and clinicopathological factors}

As Table 4 demonstrates, a positive association between the dichotomized NLR and Neu was found, with a kappa value of $0.487(P<0.0001)$. Also, in Table 4 shows, a negative correlation between the dichotomized NLR and Lym was consistently explored, with a kappa value of $0.310(P<0.0001)$. Besides, we also found that there were positive correlations between bone metastatic and pretreatment Neu\%, NLR. ( $P=0.007, P=0.022$, respectively) There were negative correlations between pretreatment $L y m \%$ and bone metastatic. $(P=0.029)$ The absolute pre-Lym tend to be correlated with PFS. $(p=0.007)$ Also, there were no correlations between other blood biomarkers with metastatic sites. We did not find the relationship between peripheral blood biomarkers and primary tumor location.

\subsection{Prognostic factor for PFS}


The median PFS were significantly related to the pre-treatment Lym, Lym\%, Neu, Neu\% and NLR. In summary, the PFS of HLym (higher in absolute lymphocyte counts) and HLym\% (higher in relative lymphocyte counts) groups were significantly higher than those of the LLym and LLym\% groups $(P<0.05$; Fig. $1 \mathrm{~A}, \mathrm{~B})$. In contrast, patients with $\mathrm{HNeu}, \mathrm{HNeu} \%$ and HNLR had poorer PFS than those with LNeu, LNeu\% and LNLR ( $P<0.05$; Fig. 1C, D; Fig.2). We also found that peripheral-type lung neoplasm has a better clinical outcome than central-type $(P=0.003, \mathrm{HR} 1.739,95 \% \mathrm{Cl} 1.207-2.506)$ (Fig2). Pre-treatment blood test were found to be important for PFS times: patients with absolute lymphocyte $<1.625$ had shorter PFS compared with $\geq 1.625$ : 13.0 months and 16.5 months $(P=0.012, H R 0.662,95 \% 0.480$ 0.915). Median PFS was 8.8 months in cases with $<18.8 \%$, while the PFS was 15.3 months in cases with $\geq 18.8 \%$. (Pख0.001, HR 0.391, 95\% $\mathrm{Cl} 0.274-0.560$ ) Besides, patients with higher neutrophil counts, relative neutrophil counts and lower NLR had a worse clinical benefit. ( $P=0.015, H R 1.481,95 \% \mathrm{Cl} 1.081-2.030$; $\mathrm{P}=0.003$, HR 1.824, HR 1.223-2.720; P凶0.001, HR 4.996, 95\%Cl 3.189-7.826). We also did not find the association between EGFR mutation and PFS $(P=0.368)$. The EGFR mutation mainly include del19 and L858R. The others are few uncommon mutations.

We used multivariate Cox regression to evaluate the independent prognostic predictors. Cox regression analysis demonstrated that NLR and, primary location that were related with PFS, which can be considered as independent factors predicting poor prognosis. (HR, 3.297, 95\% Cl 1.614-6.737, $\mathrm{P}=0.001$; $\mathrm{HR}, 2.021,95 \% \mathrm{Cl}$ 1.365-2.993 Pख0.001; respectively)

\section{Discussion}

Systemic inflammation plays a critical role in tumor proliferation and metastasis. Our study demonstrated that pretreatment neutrophil-to-lymphocyte ratio $(\geq 4.965)$ and primary location were independently and significantly associated with the shorter PFS of patients in EGFR mutant metastatic NSCLC disease. In univariate analysis, NLR, LYM, LYM\%, NEU, and NEU\% all play an important role. Before the NLR appears, the related and effective prognostic factors are known as tumor size, sex, disease status, the location of the tumor and performance status[2]. As the NLR as inflammatory marker is playing more and more influential role, it is widely analyzed in the solid tumor, such as ovarian cancer, urothelial carcinoma, head and neck cancer, lung cancer, hepatocellular carcinoma and so on[16]. The majority of the analysis focus on the multiple comprehensive treatments, including chemotherapy, chemoradiotherapy, radiation therapy, surgery, immunotherapy and immunotherapy combined radiotherapy[13,17-22]. The mechanism reflects the patients' inflammatory and systemic immune status. However, in a subset of EGFR mutant advanced disease, NLR was an important factor to assess the prognosis when treated with chemotherapy as first line. There are few studies that patients treated with targeted therapies, in particular, the EGFR-TKIs. Only few studies showed that the NLR was a significant prognostic factor for PFS in the patients who received TKI therapies. Our studies make a complementary in this field[15, 23].

To the best of our knowledge, inflammation can be regarded as the hallmark of cancer, which play an integral role in tumorigenesis, lymphomagenesis and progression of cancer ${ }^{9}$. More and more evidence 
show that elevated inflammation has the relevance of the poor cancer-specific in variety of tumors ${ }^{21}$. Tumor cells can lead to up-regulation of the inflammatory process, which can release the proinflammatory factors, inducing the promoting the cancer cells proliferation and promoting the angiogenesis and lymphagionesis. The inflammatory cells and factors, including the lymphocytes, neutrophils, platelet, IL-6, IL-8 and C-reactive protein (CRP) have different influential in various cancers [24-26]. Neutrophils and macrophage can secret the tumor growth factors, like the TL-4, IL-8 and VEGF, which can stimulate the tumor microenvironment. Yosuke Morizawa et al made analysis to detect the correlation of tumor microenvironment and neutrophil-to-lymphocyte ratio in blood in muscle bladder cancer, particular in immune cells and cytokines. They find that preoperative NLR associated with immunohischemical expression Foxp3 in bladder cancer. Besides, they also suggest that IL- 6 and IL-8 produced by cancer cells influence the level of NLR in patients with bladder cancer[25]. The same conclusion can also be found in head and neck squamous cell carcinoma, Ming-Shao Tsai et al demonstrated that NLR was positively related with level of IL-6 and PD-L1 expression[26]. Lymphocyte, especially the tumor-infiltrating lymphocytes (TILS) have a significant effect not only on the lung cancer but also in the other solid tumors.

In NSCLC, TILs play a significant role in the response the anti- PD-1 therapy in patients with metastatic. The more activated CD8+, the tumor can be controlled more better by cytotoxic activity and inducing apoptosis of cancers cells[27]. The lymphocyte counts are also used to assess the prognosis in many lung tumors. In recent analysis, the cut-off value for treatment-induced was $₫ 1,000$ cells/ $\nabla$ to evaluate the clinical benefits when patients treated with immunotherapy combining radiotherapy(RT) [28]. The preoperative lymphocyte counts is considered to be favorable prognostic factor in non-small cell lung cancer to predict the disease-free survival[27]. In our study, we find that the percentage of the lymphocyte counts play a significant role in the PFS. The elevated relative lymphocyte counts, the better clinical benefits patients receive from 1-3 lines.

In the inflammatory response to cancer, neutrophils may play a role as reservoirs for circulating vascular endothelial growth factor and promote metastasis. Previous studies have shown that the circulating neutrophils release various inflammatory factors to promote tumor progression, including factor- $a$ and interleukin-6. In our study, we also found that the higher absolute neutrophil counts and relative neutrophil counts had a shorter PFS.

Leukocytes include lymphocytes, monocytes, neutrophils, eosinophils and basophils. NLR would be a simple, inexpensive and reliable pretreatment prognostic factor for patients treated with TKIs. Iseki et al showed that LYM\% was affected by neutrophils and monocytes, which is the reason why LYM\% reflects systemic inflammation more accurately than absolute lymphocyte counts[29]. The results are consisted with our conclusion in univariate analysis. Previous analysis shows that NLR can be used as an independent prognostic factor when patients receive gefitinib or erlotinib as the first-line or second-line treatment[22]. Multivariate Cox regression show that higher pretreatment NLR was associated with worse PFS. Besides, univariate analysis demonstrated that lower baseline NLR associated with better prognosis 
in EGFR mutant metastatic NSCLC. The prognostic value of pretreatment NLR need more further prospective investigations with adequate samples to understand.

Our retrospective study supports the previous studies that the NLR is a significant factor for prognosis in NSCLC. Additionally, our reports are the first to demonstrate that NLR and primary location can be both regarded as important prognostic factors in EGFR mutant advanced NSCLC as 1-3 line treatments. More and more findings have showed that primary tumor location is one of the determined factors for choosing the optimal treatment and prognosis for patients with an advanced tumor. In our study, we use definitions according to previous findings in CT and bronchus. Virtually, peripheral adenocarcinoma had a high portion of patients in clinical benefit compared with central adenocarcinoma. Wang et al has investigated that central adenocarcinoma has a worse prognosis compared with central adenocarcinoma, which consistent with our conclusions[6]. EGFR mutation status can be also considered as prognostic factor for treatment of TKIs as first line in advanced NSCLC. According to previous analysis, patients with EGFR exon 19 deletions had longer PFS compared EGFR exon 21 mutations. Jiang et al concluded that EGFR mutation is a good predictor for patients treated with EGFR-TKIs in NSCLC[30]. A big meta-analysis also revealed that patients with EGFR exon 21 mutations had a shorter PFS compared with patients with exon 19 deletions in first line TKIs[31]. However, in our research, we compared three different EGFR status and did not draw the same conclusion. We concluded that the NLR and tumor locations are both predictive factors for the efficacy of EGFR-TKIs from first line to third line.

We are aware that there are some limitations in our analysis. First as a retrospective study, we have some selection bias. Although patients' data concerning laboratory, CT scans/PET-CT and survival data are complete, there are also a patients' selection bias. Third, the relatively numbers of eligible patients are small. In summary, the lower the percentage of lymphocytes and higher NLR, the poorer prognosis in patients treated TKIs in NSCLC. The neutrophil-to-lymphocyte ratio, and peripheral-type tumor seem clinical meaningfully for patients treated with EGFR-TKIs. As an effective and prognostic biomarker, NLR is cheap and available. We need further investigations with a large prospective study to validate our results in the future.

\section{Conclusion}

The neutrophil-to-lymphocyte ratio and primary location are both effective and meaningful factors for EGFR mutant advanced lung cancer. The primary tumor location is also a significant predictor to decide treatment planning. We also find that NLR was a useful predictor for systemic inflammation in patients treated with TKIs. However, we need more data to explore understand the relationship among parameters. Our findings support the existing hypothesis that systemic inflammation is associated with clinical outcomes.

\section{Declarations}

Ethics approval and consent to participate 
The studies involving human participants were reviewed and approved by The Ethics Committee of

Shandong Cancer Hospital Affiliated to Shandong First Medical University. The patients/participants provided agree to participate in this study.

Consent for publication

Not applicable.

Availability of data and materials

Al the data and material supporting the findings are present in the manuscript.

Competing interests

There is no conflict of interest among authors to disclose.

Funding

This project was support by the National Natural Science Foundation of China (81502667), Key Research and Development Plan of Shandong, China (2016GSF201167), and Natural Science Foundation of Shandong, China (2019PH111). The funding bodies had role in the study design and data collection.

Authors' contributions

Writing - original draft: XQX; Writing - review and editing: JMY, MHL and PX; Conceptualization: PX, XLL, WJT; Data collection and analysis: WJT, JLC; All authors have read and approved the final manuscript.

Acknowledgements

The authors would like to acknowledge Department of Radiation Oncology, Shandong Cancer Hospital \& Institute, for his organizational support.

\section{Abbreviations}

EGFR: epidermal growth factor receptor; TKIs: tyrosine kinase inhibitors; NLR: neutrophil-to-lymphocyte ratio; PFS: progression free survival; NSCLC: non-small cell lung cancer; Lym: absolute lymphocyte count; Lym\%: lymphocyte percentage; Neu: absolute neutrophil count; Neu\%: the percentage of neutrophil granulocytes; LMR: Iymphocyte-to-monocyte ratio; ICls: Immune check point inhibitors; OS: overall survival; $\mathrm{CBC}$ : complete blood count; $\mathrm{CT}$ : Computerized tomography; PET: position emission tomography; PNA: peptide nucleic acid; PCR: Polymerase Chain Reaction; NGS: next generation sequencing; ROC: receiver operating characteristic; AUC: Area Under the Curve; HLYM: higher in absolute lymphocyte counts; HLYM\%: higher in relative lymphocyte counts; LLYM: lower in absolute lymphocyte counts; LLYM\%: lower in relative lymphocyte counts; HNEU: higher in absolute neutrophil count; HNEU\%: higher in the 
percentage of neutrophil granulocytes; HNLR: higher in NLR; LNEU: lower in absolute neutrophil count; LNEU\%: lower in the percentage of neutrophil granulocytes; LNLR: lower in NLR.

\section{References}

1. Siegel, R.L., K.D. Miller, and A. Jemal, Cancer statistics, 2020. CA Cancer J Clin, 2020. 70(1): p. 7-30.

2. Berghmans, T., M. Paesmans, and J.P. Sculier, Prognostic factors in stage III non-small cell lung cancer: a review of conventional, metabolic and new biological variables. Ther Adv Med Oncol, 2011. 3(3): p. 127-38.

3. Sun, W., et al., Primary Tumor Location Is a Useful Predictor for Lymph Node Metastasis and Prognosis in Lung Adenocarcinoma. Clin Lung Cancer, 2017. 18(1): p. e49-e55.

4. Moon, Y., et al., Differing histopathology and prognosis in pulmonary adenocarcinoma at central and peripheral locations. J Thorac Dis, 2016. 8(1): p. 169-77.

5. Park, H.S., et al., Central versus Peripheral Tumor Location: Influence on Survival, Local Control, and Toxicity Following Stereotactic Body Radiotherapy for Primary Non-Small-Cell Lung Cancer. J Thorac Oncol, 2015. 10(5): p. 832-837.

6. Wang, Z., et al., Primary tumor location is an important predictor of survival in pulmonary adenocarcinoma. Cancer Manag Res, 2019. 11: p. 2269-2280.

7. Kim, J. and J.S. Bae, Tumor-Associated Macrophages and Neutrophils in Tumor Microenvironment. Mediators Inflamm, 2016. 2016: p. 6058147.

8. Helm, 0., et al., Tumor-associated macrophages exhibit pro- and anti-inflammatory properties by which they impact on pancreatic tumorigenesis. Int J Cancer, 2014. 135(4): p. 843-61.

9. Noy, R. and J.W. Pollard, Tumor-associated macrophages: from mechanisms to therapy. Immunity, 2014. 41(1): p. 49-61.

10. Hanahan, D. and R.A. Weinberg, Hallmarks of cancer: the next generation. Cell, 2011. 144(5): p. 64674.

11. Prelaj, A., et al., EPSILoN: A Prognostic Score Using Clinical and Blood Biomarkers in Advanced NonSmall-cell Lung Cancer Treated With Immunotherapy. Clin Lung Cancer, 2020.

12. Castello, A., et al., The immune-metabolic-prognostic index and clinical outcomes in patients with non-small cell lung carcinoma under checkpoint inhibitors. J Cancer Res Clin Oncol, 2020. 146(5): p. 1235-1243.

13. Diem, S., et al., Neutrophil-to-Lymphocyte ratio (NLR) and Platelet-to-Lymphocyte ratio (PLR) as prognostic markers in patients with non-small cell lung cancer (NSCLC) treated with nivolumab. Lung Cancer, 2017. 111: p. 176-181.

14. Mandaliya, $\mathrm{H}$., et al., Prognostic biomarkers in stage IV non-small cell lung cancer (NSCLC): neutrophil to lymphocyte ratio (NLR), lymphocyte to monocyte ratio ( $L M R)$, platelet to lymphocyte ratio (PLR) and advanced lung cancer inflammation index (ALI). Transl Lung Cancer Res, 2019. 8(6): p. 886-894. 
15. Ono, T., et al., Impact of neutrophil-to-lymphocyte ratio in patients with EGFR-mutant NSCLC treated with tyrosine kinase inhibitors. Invest New Drugs, 2020. 38(3): p. 885-893.

16. Templeton, A.J., et al., Prognostic role of neutrophil-to-lymphocyte ratio in solid tumors: a systematic review and meta-analysis. J Natl Cancer Inst, 2014. 106(6): p. dju124.

17. Kiriu, T., et al., Pseudo-Progression and the Neutrophil-to-Lymphocyte Ratio in Non-Small Cell Lung Cancer Treated with Immune Checkpoint Inhibitors: A Case-Control Study. Onco Targets Ther, 2019. 12: p. 10559-10568.

18. Hasegawa, T., et al., Association of High Neutrophil-to-Lymphocyte Ratio With Poor Outcomes of Pembrolizumab Therapy in High-PD-L 1-expressing Non-small Cell Lung Cancer. Anticancer Res, 2019. 39(12): p. 6851-6857.

19. Xia, L.J., et al., Significance of neutrophil-to-lymphocyte ratio, platelet-to-lymphocyte ratio, lymphocyte-to-monocyte ratio and prognostic nutritional index for predicting clinical outcomes in T12 rectal cancer. BMC Cancer, 2020. 20(1): p. 208.

20. Lin, G.N., et al., Elevated neutrophil-to-lymphocyte ratio predicts poor outcome in patients with advanced non-small-cell lung cancer receiving first-line gefitinib or erlotinib treatment. Asia Pac J Clin Oncol, 2017. 13(5): p. e189-e194.

21. Meriggi, F., et al., Significance of neutrophil-to-lymphocyte ratio in Western advanced EGFR-mutated non-small cell lung cancer receiving a targeted therapy. Tumori, 2017. 103(5): p. 443-448.

22. Phan, T.T., et al., The prognostic impact of neutrophil to lymphocyte ratio in advanced non-small cell lung cancer patients treated with EGFR TKI. Int J Gen Med, 2018. 11: p. 423-430.

23. Aguiar-Bujanda, D., et al., Neutrophil to Lymphocyte Ratio as a Prognostic Factor in European Patients with Epidermal Growth Factor Receptor-Mutant Non-Small Cell Lung Cancer Treated with Tyrosine Kinase Inhibitors. Oncol Res Treat, 2018. 41(12): p. 755-761.

24. Nakamura, T., et al., The combined use of the neutrophil-lymphocyte ratio and C-reactive protein level as prognostic predictors in adult patients with soft tissue sarcoma. J Surg Oncol, 2013. 108(7): p. 481-5.

25. Morizawa, Y., et al., Correlation of Immune Cells and Cytokines in the Tumor Microenvironment with Elevated Neutrophil-To-Lymphocyte Ratio in Blood: An Analysis of Muscle-Invasive Bladder Cancer. Cancer Invest, 2018. 36(7): p. 395-405.

26. Tsai, M.S., et al., The prognosis of head and neck squamous cell carcinoma related to immunosuppressive tumor microenvironment regulated by IL-6 signaling. Oral Oncol, 2019. 91: p. 4755.

27. Zhang, J., et al., Preoperative lymphocyte count is a favorable prognostic factor of disease-free survival in non-small-cell lung cancer. Med Oncol, 2013. 30(1): p. 352.

28. Cho, Y., et al., Impact of Treatment-Related Lymphopenia on Immunotherapy for Advanced Non-Small Cell Lung Cancer. Int J Radiat Oncol Biol Phys, 2019. 105(5): p. 1065-1073.

29. Iseki, Y., et al., The impact of the preoperative peripheral lymphocyte count and lymphocyte percentage in patients with colorectal cancer. Surg Today, 2017. 47(6): p. 743-754. 
30. Jiang, H., et al., Association between EGFR exon 19 or exon 21 mutations and survival rates after first-line EGFR-TKI treatment in patients with non-small cell lung cancer. Mol Clin Oncol, 2019. 11(3): p. 301-308.

31. Zhang, Y., et al., Patients with exon 19 deletion were associated with longer progression-free survival compared to those with L858R mutation after first-line EGFR-TKIs for advanced non-small cell lung cancer: a meta-analysis. PLoS One, 2014. 9(9): p. e107161.

\section{Tables}

Table 1 Patients and clinical characteristics

\begin{tabular}{|c|c|}
\hline Characteristics & $\begin{array}{l}\text { All patients }(\mathrm{n}=190) \\
\mathrm{n}(\%)\end{array}$ \\
\hline \multicolumn{2}{|l|}{ Gender } \\
\hline Male & $68(35.8 \%)$ \\
\hline Female & $122(64.2 \%)$ \\
\hline \multicolumn{2}{|l|}{ Age } \\
\hline Median & 58 \\
\hline Range & $34-87$ \\
\hline \multicolumn{2}{|l|}{ Smoking habits } \\
\hline Non-smoker & $148(77.9 \%)$ \\
\hline Ever-smoker & $42(22.1 \%)$ \\
\hline \multicolumn{2}{|l|}{ EGFR-mutation } \\
\hline L858R & $110(57.9 \%)$ \\
\hline $19 \mathrm{del}$ & $75(39.5 \%)$ \\
\hline Rare mutation & $5(2.6 \%)$ \\
\hline \multicolumn{2}{|l|}{ Stage } \\
\hline Postoperative recurrence & $26(13.7 \%)$ \\
\hline IV & $164(86.3 \%)$ \\
\hline \multicolumn{2}{|l|}{ EGFR Mutation Test } \\
\hline PCR & $172(90.5 \%)$ \\
\hline NGS & $18(9.5 \%)$ \\
\hline \multicolumn{2}{|l|}{ Tumor location } \\
\hline Central type & $48(25.3 \%)$ \\
\hline Peripheral type & $142(74.7 \%)$ \\
\hline \multicolumn{2}{|l|}{ Bone metastasis } \\
\hline YES & $83(43.7 \%)$ \\
\hline NO & $107(56.3 \%)$ \\
\hline \multicolumn{2}{|l|}{ Liver metastasis } \\
\hline YES & $26(13.7 \%)$ \\
\hline NO & $164(86.3 \%)$ \\
\hline \multicolumn{2}{|l|}{ Brain metastasis } \\
\hline YES & $59(31.1 \%)$ \\
\hline NO & $131(68.9 \%)$ \\
\hline \multicolumn{2}{|l|}{ First-line therapy } \\
\hline Surgery & $27(14.2 \%)$ \\
\hline Chemotherapy & $93(49.0 \%)$ \\
\hline Chemoradiotherapy & $9(4.7 \%)$ \\
\hline Radiotherapy combined targeted therapy & $15(7.9 \%)$ \\
\hline Targeted therapy & $36(18.9 \%)$ \\
\hline Radiotherapy & $6(3.2 \%)$ \\
\hline Chemotherapy combined with TKIs & $4(2.1 \%)$ \\
\hline \multicolumn{2}{|l|}{ Target therapy lines } \\
\hline First-line & $54(28.4 \%)$ \\
\hline Second-line & $102(53.7 \%)$ \\
\hline Third-line & $34(17.9 \%)$ \\
\hline
\end{tabular}


Table 2

Univariate and multivariate Cox proportional analysis regarding PFS $(n=190)$

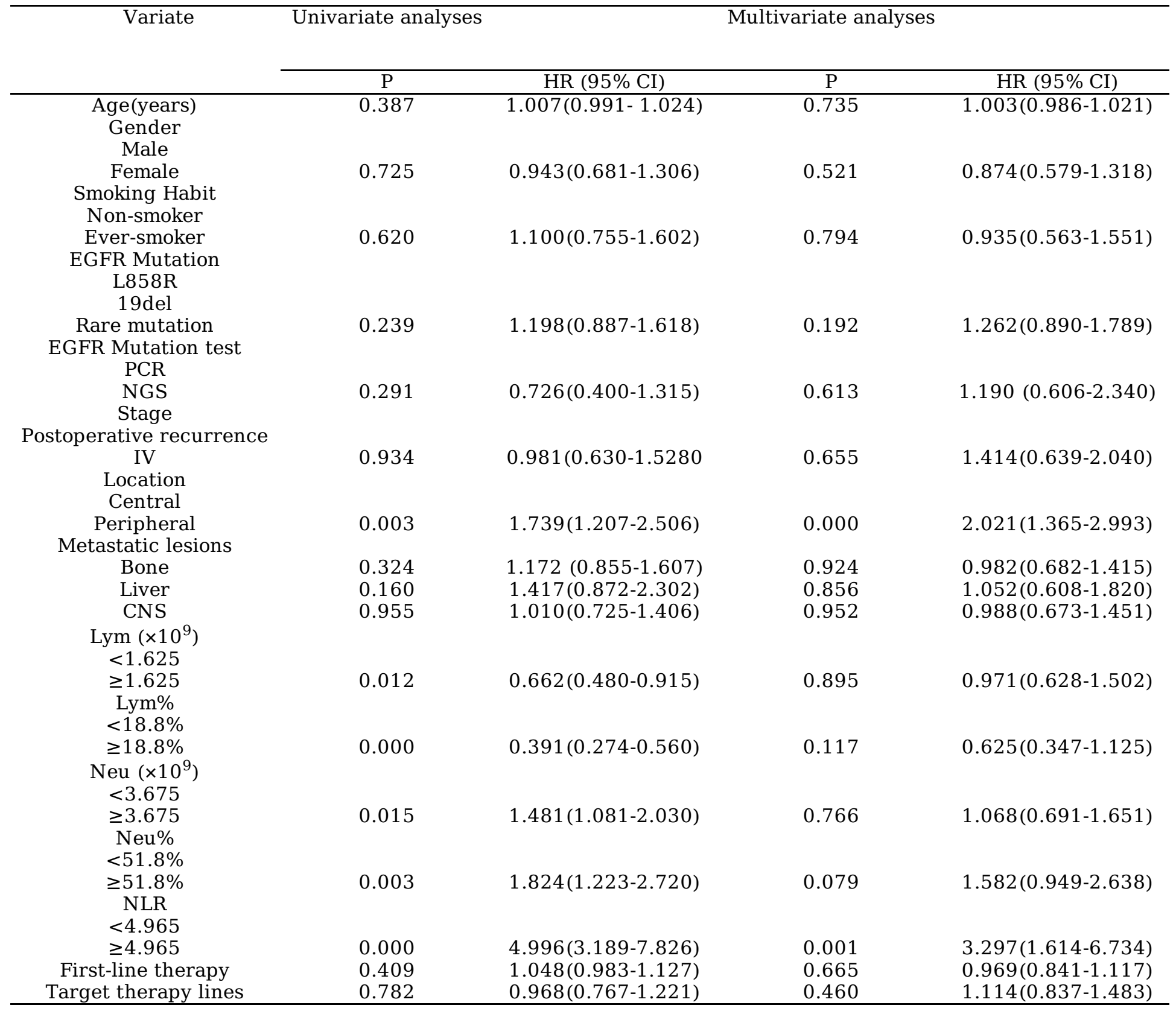

Table 3 The optimal cut-off values based on PFS

\begin{tabular}{llll}
\hline Peripheral blood index & Sensitivity & Specificity & AUC \\
\hline Lym $\left(\times 10^{9} / \mathrm{L}\right)$ & $63 \%$ & $64.4 \%$ & 0.632 \\
Lym $\%$ & $88.9 \%$ & $28.2 \%$ & 0.549 \\
Neu $\left(\times 10^{9} / \mathrm{L}\right)$ & $59.3 \%$ & $54 \%$ & 0.521 \\
Neu $\%$ & $88.9 \%$ & $19 \%$ & 0.499 \\
NLR & $17.8 \%$ & $100.0 \%$ & 0.550 \\
\hline
\end{tabular}


Table 4 Peripheral blood test with clinicopathological factors with $\mathrm{P}$ values

\begin{tabular}{lllll}
\hline Parameter & R spearman & P value & U test & P value \\
\hline NLR\& Lym & -0.310 & 0.000 & & \\
NLR\& Neu & 0.595 & 0.000 & & \\
Lym\%\& Bone metastatic & & & 3617.500 & 0.029 \\
Neu\%\&Bone metastatic & & & 5442.5 & 0.008 \\
NLR\&Bone metastatic & & 5299.5 & 0.022 \\
\hline
\end{tabular}

Figures
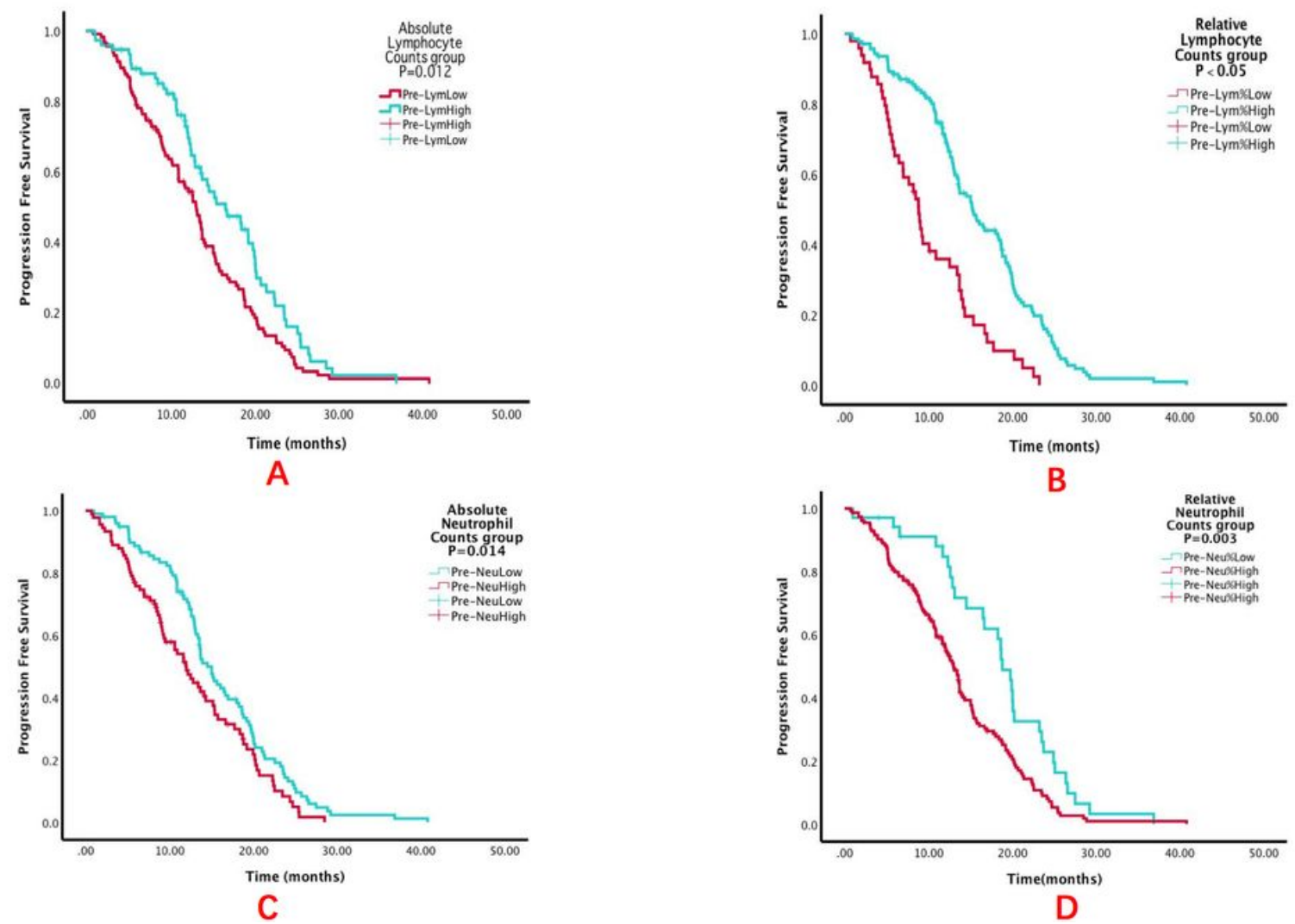

Figure 1

Kaplan-Meier plot of progression-free survival (PFS) stratified by pretreatment absolute lymphocyte counts group $(A)$, relative lymphocyte counts group (B), absolute neutrophil counts group (C), and relative neutrophil counts group (D). 


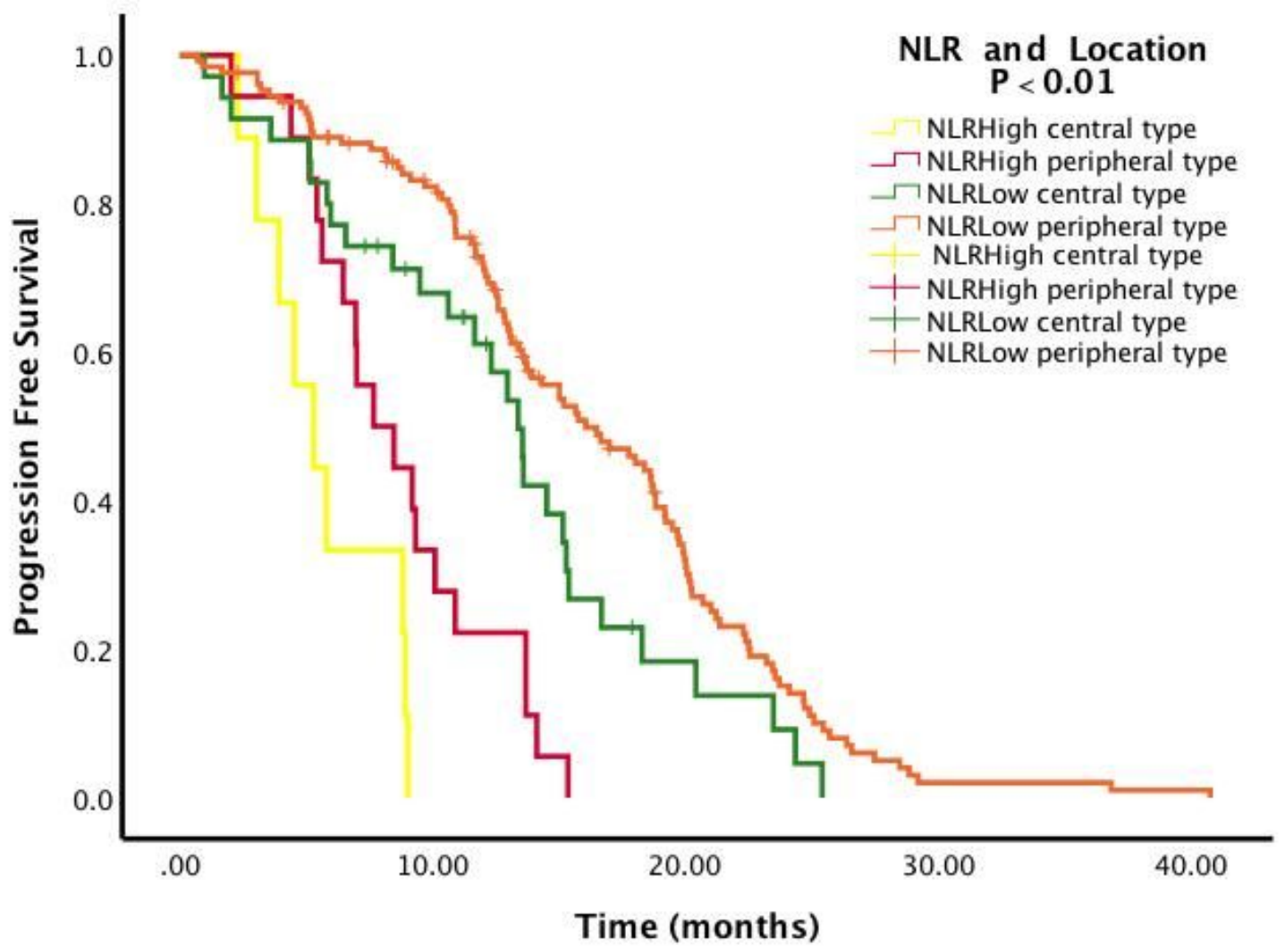

Figure 2

Kaplan-Meier survival curves of PFS based on combination of pretreatment neutrophil to lymphocyte expression and primary location in all patients.
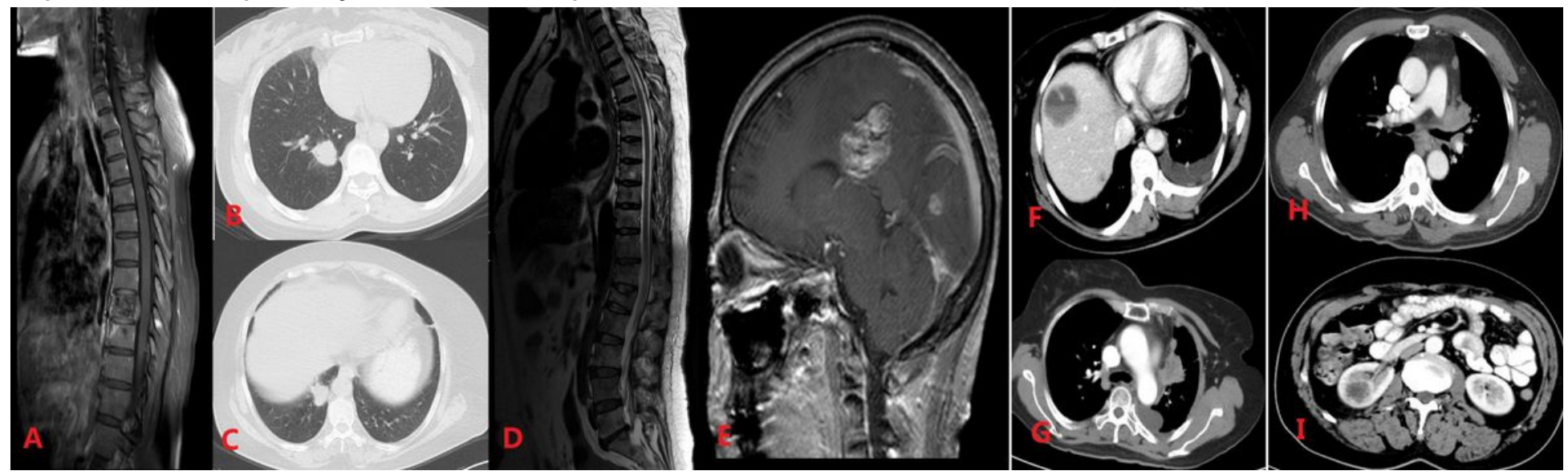

Figure 3

A patient with high NLR and peripheral type (B) in stage IV non-small cell lung cancer (A) with shorter PFS. A patient with low NLR and peripheral type (C) in stage IV lung adenocarcinoma with longer PFS. (D) A patient with low NLR and central (G) in stage IV lung adenocarcinoma (E, F) with shorter PFS. A patient with high NLR and central type $(\mathrm{H})$ in stage IV (I) with longer PFS. 